\title{
Persistent left superior vena cava: A possible contraindication to chemotherapy and total parenteral nutrition in cancer patients
}

\author{
FRANCESCO IOVINO $^{1}$, PASQUALE PIO AURIEMMA ${ }^{1}$, LUCA DEL VISCOVO ${ }^{2}$, \\ SARA SCAGLIARINI ${ }^{3}$, MARILENA DI NAPOLI ${ }^{3}$ and FERDINANDO DE VITA ${ }^{3}$ \\ ${ }^{1}$ Division of General Surgery, Department of Anesthesiological, Surgical and Emergency Sciences; \\ Divisions of ${ }^{2}$ Radiology and ${ }^{3}$ Medical Oncology, 'F. Magrassi - A. Lanzara' \\ Department of Clinical and Experimental Medicine and Surgery, Second University of Naples, Naples, Italy
}

Received April 2, 2012; Accepted July 5, 2012

DOI: $10.3892 / \mathrm{ol} .2012 .808$

\begin{abstract}
Persistent left superior vena cava (PLSVC) is the most common thoracic venous anomaly. Awareness of this condition may be useful when placement of left-side transvenous subclavian or internal jugular catheters is required. This anomaly may be detected only by chest radiograph following placement of the catheter. The primary endpoints of this study were to analyze the prevalence of PLSVC, measurement of its diameters and the outcome of cancer patients with this anomaly undergoing placement of a long term catheter for nutrition and chemotherapy at the Department of Surgery, of the Second University of Naples, Naples, Italy. A total of 600 consecutive adult patients with hematological or solid tumors admitted to our surgery department for implantation of a central venous catheter (CVC) were considered. The CVC was routinely implanted in the left internal jugular vein under ultrasound guidance. Four cases of PLSVC ( $0.6 \%$ of patients) were observed and confirmed using cine magnetic resonance imaging (MRI). In all cases, the CVC was not removed. Three patients underwent chemotherapy and one patient was subjected to total parenteral nutrition. In the three patients undergoing chemotherapy, dynamic ECG and echocardiography were performed at the end of the treatment. No disturbances of the cardiac rhythm or thrombosis were detected, and heart ejection fraction (EF) was not affected. In conclusion, although PLSVC may be a risky condition, no complications occurred in our study. Thus, PLSVC should not be regarded as a strict contraindication to infusion of chemotherapy or hyperosmolar nutritional solutions. However, further research is needed to confirm our data.
\end{abstract}

Correspondence to: Professor Francesco Iovino, Division of General Surgery, Department of Anesthesiological, Surgical and Emergency Sciences, Second University of Naples, Piazza Miraglia 1, Naples, Italy

E-mail: francesco.iovino@unina2.it

Key words: central venous catheter, persistent left superior vena cava, cancer patients

\section{Introduction}

Persistent left superior vena cava (PLSVC) is the most common thoracic venous anomaly (1). PLSVC is present in $0.3-0.5 \%$ of the general population $(2,3)$. It is usually asymptomatic and hemodynamically irrelevant or associated with disturbances of cardiac rhythm. Awareness of this condition may be useful when placement of left-side transvenous subclavian or internal jugular catheters is required, in such settings as critical care, nefrology, onco-hematology and anaesthesiology (4). Ultrasound-guided central vascular access is an emerging application which offers the advantage of a shorter access time and a reduced number of attempts compared with the landmark-guided technique (5), but is not useful in identifying PLSVC. Therefore, this anomaly may be detected only by chest X-ray during catheterization or following placement of a catheter (6). In the present study, we report the prevalence of PLSVC, its diameters and the outcome of cancer patients with this anomaly undergoing placement of a long-term catheter for nutrition and chemotherapy at our institution.

\section{Materials and methods}

Catheter placement. Adult patients with hematological or solid tumors admitted to our surgery department for implantation of a central venous catheter (CVC) were considered. The study was approved by the ethics committee of the Second University of Naples, Naples, Italy. Informed consent was obtained from each patient prior to the study. All procedures were performed by the same surgeon with specific experience in ultrasound guided catheterization. The CVC was routinely implanted in the left internal jugular vein; however, if conditions were unsuitable for implantation on this side, such as in the case of lymphadenopathy or postradiation therapy, or on patient request, the CVC was placed on the right side. Ultrasound examinations were performed using ESAOTE (Genova, Italy), equipped with a $7.5 \mathrm{mHz}$ probe. Patients were placed in the Trendelenburg position with the head rotated toward the opposite side. All procedures were performed using standard aseptic techniques and local anesthesia. Puncture of the internal jugular vein was applied to the last portion $1 \mathrm{~cm}$ above the clavicle and behind the clavicular head of the 
sternocleidomastoid muscle (7). Correct venipuncture was always confirmed by ultrasound guidance and easy aspiration of venous blood. The Seldinger technique, using a peel-away sheath, was used to place the catheter into the superior vena cava until insertion into the right atrium.

In all cases, at the end of the procedure, a chest radiograph in double projection was carried out to verify the correct placement of the catheter. In case of suspected PLSVC, a cine magnetic resonance imaging (MRI) scan was always performed. The patients' demographic data, diagnosis, indications for CVC insertion, type of CVC, side of venipuncture, number of attempts, time of procedure, malposition of the catheter and early complications were recorded for all procedures. Systemic prophylaxis against deep-vein thrombosis was adopted for 20 days and no antibiotic prophylaxis was prescribed. Follow-up for each patient with clinical examination and ultrasound exploration of the vein was scheduled after 30 days.

Data analysis. Demographic data and clinical features were analyzed using descriptive methods. Quantitative variables were summarized using the mean and standard deviations (SD). Categorical variables were summarized as counts and percentages.

\section{Results}

From July 2005 to December 2010, 600 patients with hematological or solid tumors underwent central venous catheterization (CVC). Indications for CVC were chemotherapy (86.6\%), transfusion $(0.8 \%)$, parenteral nutrition $(8.3 \%)$ and palliative care $(4.1 \%)$. The group comprised of 420 females $(80 \%)$ and 120 males $(20 \%)$, with a mean age of 58 years (range, 15-75) (Table I). The type of catheter, side and site of venipuncture are shown in Table II. Four cases of PLSVC $(0.6 \%)$ were suspected based on chest radiography findings (Fig. 1). Cine MRI confirmed an isolated PLSVC in one patient and a PLSVC associated with a right superior vena cava (RSVC) in other three cases (Fig. 2). The mean diameter \pm SD of the PLSVC was $14.77 \pm 1.15 \mathrm{~mm}$. In all cases, the CVC was not removed. Three patients underwent chemotherapy and one patient was subjected to total parenteral nutrition (TPN) (Table III). A dynamic ECG was performed before starting and during the first cycle of chemotherapy in all patients. In the three patients undergoing chemotherapy, dynamic ECG and echocardiography were repeated at the end of treatment. No disturbances in cardiac rhythm were noted and the heart ejection fraction (EF) was not affected (Table III). The patient on TPN succumbed to pancreatic cancer progression after two months. For the other patients, no evidence of infection or malfunction of the catheters was observed in the following outpatient visits.

\section{Discussion}

The prevalence of PLSVC is $0.3-0.5 \%$ in the general population. In $80-90 \%$ of individuals, PLSVC drains into the right atrium directly or via the coronary sinus and is of no hemodynamic consequence. In the remaining cases, it may drain into the left atrium, resulting in a right to left sided shunt (1-4). Almost $40 \%$ of patients with PLSVC have a variety of associated cardiac anomalies, such as atrial septal defects, bicuspid
Table I. Patient characteristics.

\begin{tabular}{lcr}
\hline Characteristic & No. of patients & $\%$ \\
\hline Total & 600 & 100.0 \\
Age (years), mean (range) & $58(15-75)$ & \\
Gender & & \\
Male & 120 & 20.0 \\
Female & 480 & 80.0 \\
Treatment & & \\
Chemotherapy & 520 & 86.6 \\
Total parenteral nutrition & 50 & 4.1 \\
Palliative care & 25 & 0.8 \\
Transfusion & 5 & \\
\hline
\end{tabular}

Table II. Type of catheter implanted and side of venipuncture.

\begin{tabular}{lcc}
\hline & $\begin{array}{c}\text { No. of } \\
\text { patients }\end{array}$ & $\%$ \\
\hline Totally implantable catheter 7 Fr & 480 & 80 \\
Tunneled external catheter 7 Fr & 120 & 20 \\
Internal jugular left side & 510 & 85 \\
Internal jugular right side & 90 & 15 \\
\hline
\end{tabular}

aortic valve, coarctation of the aorta, coronary sinus ostial atresia and cor triatrium $(9,10)$. The diagnosis of PLSVC is usually made as an incidental finding during cardiovascular imaging or placement of a central venous catheter via the left jugular or subclavian vein $(3,4)$. Chest X-ray demonstrates an unusual course of the catheter in the left hemithorax. A CT scan or MRI may be employed to establish the diagnosis. Echocardiography is useful to verify the presence of a dilated coronary sinus and to rule out variations in the typical anomalous venous course $(2,8,10,11)$. In our group of patients, the prevalence of PLSVC was more elevated than in the general population, possibly owing to the choice of inserting the catheter on the left side. In the four cases of suspected PLSVC on chest radiograph, a cine MRI was carried out in order to study both the path of the PLSVC and the blood vessels. Three patients had both PLSVC and RSVC. Agenesia of RSVC and presence of PLSVC were confirmed by cine MRI in only one patient. Since echocardiography was performed before starting chemotherapy, it was not repeated. PLSVC has various practical implications when the left veins are used to access the right side of the heart or pulmonary vasculature. Arrhythmia, cardiogenic shock, cardiac tamponade and coronary sinus thrombosis have been reported when catheters have been inserted via PLSVC. Fortunately, the incidence of such complications is relatively low $(3,10,12,13,14)$. In cancer patients, the placement of a CVC in PLSVC may have practical implications as chemotherapic drugs and hyperosmolar solutions are injected into a smaller vein. In our patients, endothelial damage and risk of thrombosis or anomalies of the cardiac rhythm were feared. Indeed, these complications are 
Table III. Characteristics of 4 patients with PLSVC.

\begin{tabular}{|c|c|c|c|c|}
\hline Characteristic & Case 1 & Case 2 & Case 3 & Case 4 \\
\hline Age (years) & 66 & 74 & 52 & 54 \\
\hline Gender & Male & Female & Female & Female \\
\hline Solid tumor & Lung & Breast & Pancreas & Ovarian \\
\hline Indication (protocol) & $\begin{array}{c}\text { CHT } \\
\text { (Carboplatin + etoposide } \\
\text { for } 6 \text { cycles) }\end{array}$ & $\begin{array}{c}\text { CHT } \\
\text { (FEC } 4 \text { cycles }+ \\
\text { taxotere } 4 \text { cycles) }\end{array}$ & $\begin{array}{c}\text { TPN } \\
\text { (2000 Kcal/day) }\end{array}$ & $\begin{array}{c}\text { CHT } \\
\text { (Carboplatin }+ \text { taxol } \\
\text { for } 6 \text { cycles })\end{array}$ \\
\hline ECG findings & Normal & Normal & Normal & Normal \\
\hline $\mathrm{EF} \%$ pre-CHT & 60 & 55 & 66 & 60 \\
\hline Diameter of PLSVC (mm) & 14.2 & 15.3 & 16.1 & 13.5 \\
\hline External diameter of CVC (mm) & 2.31 & 2.31 & 2.31 & 2.31 \\
\hline Absence of RSVC & No & Yes & No & No \\
\hline CS dilated & No & No & No & No \\
\hline EF \% post-CHT & 60 & 55 & Died after 2 months & 60 \\
\hline $\begin{array}{l}\text { Anomalies of cardiac rhythm } \\
\text { post-CHT }\end{array}$ & No & No & No & No \\
\hline Thrombosis & No & No & No & No \\
\hline
\end{tabular}

PLSVC, persistent left superior vena cava; EF, ejection fraction; CHT, chemotherapy; TPN, total parenteral nutrition; CVC, central venous catheter; RSVC, right superior vena cava; CS, coronary sinus.

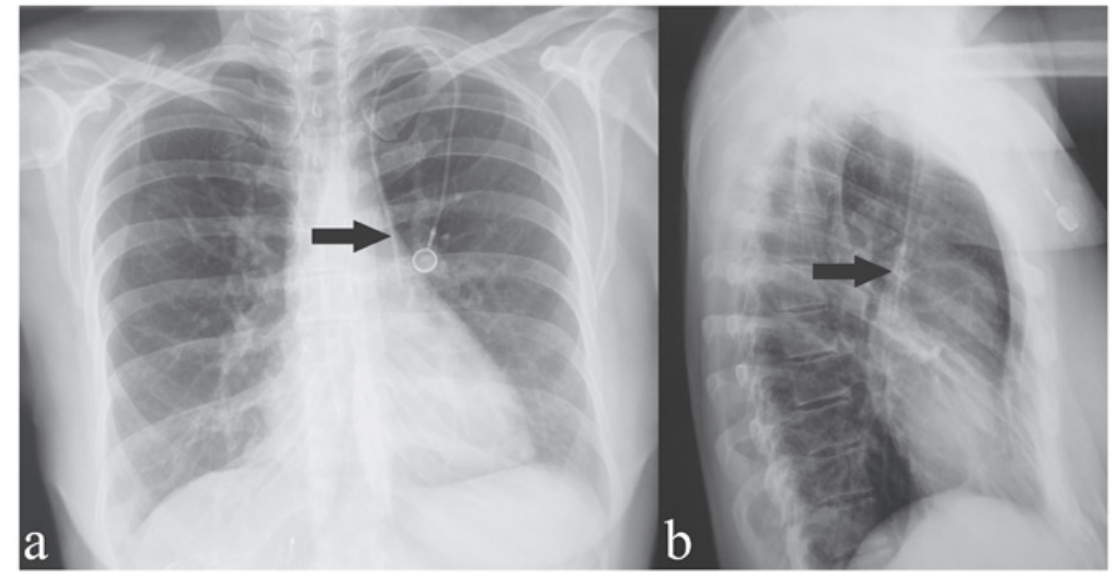

Figure 1. Chest X-ray demonstrating unusual course of catheter (arrow) in the left hemithorax, (a) AP and (b) LL view, rather than in the right side via the normal anatomical position of the superior vena cava. AP, anterior-posterior; LL, latero-lateral.

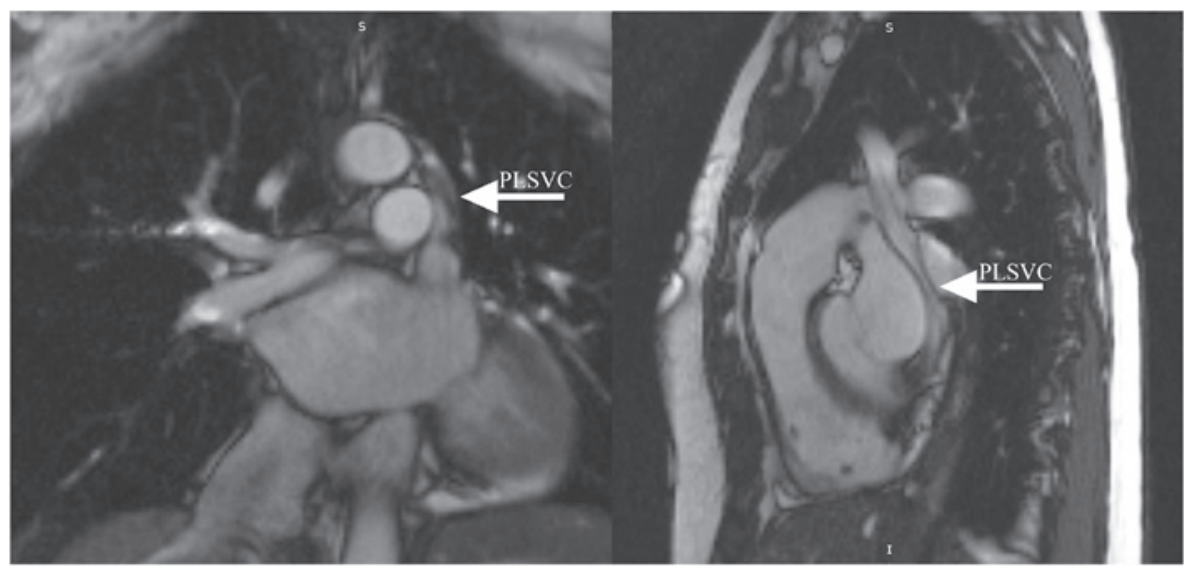

Figure 2. Cine MRI showing an isolated PLSVC (arrow), which leads directly into the right atrium. PLSVC, persistent left superior vena cava. 
more likely when the diameter of the vein is small, the blood flow is slowed or the catheter is large. In our four patients, no clinically significant complications were recorded. The mean diameter \pm SD of the PLSVC was $14.77 \pm 1.15 \mathrm{~mm}$ and the external diameter of the catheter was $7 \mathrm{Fr}(2.31 \mathrm{~mm})$. Cardiac rhythm disturbances were not observed on dynamic ECG, and structural heart damage was ruled out by echocardiography. We are unable to draw conclusions as to what would occur in the case of PLSVC draining into the coronary sinus. In conclusion, although PLSVC may be a risky condition, no complications were recorded in the present study when the CVC was positioned in PLSVC for administering chemotherapeutic drugs or hyperosmolar solutions. However, further research is needed to confirm our data.

\section{References}

1. Goyal SK, Punnam SR, Verma G and Ruberg FL: Persistent left superior vena cava: a case report and review of literature. Cardiovasc Ultrasound 6: 50, 2008.

2. Saranteas T, Mandila C, Poularas J, Papanicolaou J, Patriankos A, Karakitsos D and Karabinis A: Transesophageal echocardiography and vascular ultrasound in the diagnosis of catheter-related persistent left superior vena cava thrombosis. Eur J Echocardiogr 10: 452-455, 2009.

3. Parreira LF, Lucas CC, Gil CC and Barata JD: Catheterization of a persistent left superior vena cava. J Vasc Access 10: 214-215, 2009.

4. Pahwa R and Kumar A: Persistent left superior vena cava: an intensivist's experience and review of the literature. South Med J 96: 528-529, 2003.
5. Cavanna L, Civardi G, Vallisa D, Di Nunzio C, Capucciati L, et al: Ultrasound-guided central venous catheterization in cancer patients improves the success rate of cannulation and reduces mechanical complications: A prospective observational study of 1,978 consecutive catheterizations. World J Surg Oncol 8: 91-98, 2010.

6. Gonzales-Juanatey C, Testa A, Vidan J, et al: Persistent left superior vena cava draining into the coronary sinus: Report of 10 cases and literature review. Clin Cardiol 27: 515-518, 2004.

7. Iovino F, Pittiruti M, Buononato M and Lo Schiavo F: Central venous catheterization: complications of different placements. Ann Chir 126: 1001-1016, 2001.

8. Voci P, Luzi G and Agati L: Diagnosis of persistent left superior vena cava by multiplane transesophageal echocardiography. Cardiologia 40: 273-275, 1995.

9. Sarodia B and Stoller J: Persistent left superior vena cava: case report and literature review. Respir Care 45: 411-416, 2000.

10. Recupero A, Pugliatti P, Rizzo F, Carerj S, Cavalli G, et al: Persistent left-sided superior vena cava: integrated noninvasive diagnosis. Echocardiography 24: 982-986, 2007.

11. Kute VB, Vanikar AV, Gumber MR, Shah PR, Goplani KR and Trivedi HL: Hemodialysis through persistent left superior vena cava. Indian J Crit Care Med 15: 40-42, 2011

12. Wissner E, Tilz R, Konstantinidou M, Metzner A, Schmidt B, Chun KR, Kuck KH and Ouyang F: Catheter ablation of atrial fibrillation in patients with persistent left superior vena cava is associated with major intraprocedural complications. Heart Rhythm 7: 1755-1760, 2010.

13. Laurenzi L, Natoli S, Pelagalli L, Marcelli ME, Abbattista D, Carpanese L and Arcuri E: Long-term central venous catheterization via persistent left superior vena cava: a case report. Support Care Cancer 11: 190-192, 2003.

14. Pai RG: Echocardiographic features of persistent left superior vena cava. Echocardiography 16: 435-436, 1999. 